\title{
PRIDE contribution to the European VLBI Network
}

\author{
Guifré MOLERA CALVÉS* \\ Joint Institute for VLBI in Europe \\ Aalto University \\ E-mail: moleraejive.nl
}

\section{Giuseppe CIMÒ}

Joint Institute for VLBI in Europe

\section{Sergei POGREBENKO}

Joint Institute for VLBI in Europe

\section{Dmitry DUEV}

Joint Institute for VLBI in Europe

\section{Tatiana BOCANEGRA-BAHAMÓN}

Joint Institute for VLBI in Europe

Delft University of Technology

Shanghai Astronomical Observatory

\section{Leonid GURVITS}

Joint Institute for VLBI in Europe

Delft University of Technology

\begin{abstract}
The VLBI and Doppler tracking technique is one of the most powerful tools for determining accurately the position of a planetary spacecraft. The Planetary Radio Interferometry and Doppler Experiment (PRIDE), an initiative by the Joint Institute for VLBI in Europe (JIVE), is a multipurpose and multi-disciplinary enhancement of planetary missions science return. PRIDE provides estimates of spacecraft state vectors based on VLBI phase-reference and radial Doppler measurements.

In this paper, a brief summary of the methodology and data processing is introduced. Additionally, several scientific studies that benefit from Doppler measurements of planetary spacecraft tracking are presented. Special emphasis is on those cases that the group is currently involved.
\end{abstract}

11th European VLBI Network Symposium \& Users Meeting,

October 9-12, 2012

Bordeaux, France

${ }^{*}$ Speaker. 


\section{INTRODUCTION}

Using planetary and deep-space science mission spacecraft as targets of radio astronomy offer new tools for studying a broad variety of physical processes. These processes include the dynamics of extraterrestrial atmospheres, geodynamic diagnostics of the interior of planets and structures of the satellite rings, fundamental physics, effects of the spacecraft motion, studies of the solar corona, and characterization of the solar wind. Many of these applications require an extremely high angular resolution coupled with very high spectral resolution, only achievable with VLBI. The spacecraft VLBI tracking technique has been already successfully validated in several experiments, among others, the VLBI tracking of the descent and landing of Huygens probe in the atmosphere of Titan [1].

Planetary Radio Interferometry and Doppler Experiment (PRIDE) is the latest development in radio science based on VLBI spacecraft tracking. PRIDE is adopted by a number of prospective planetary science missions as a port of their scientific suite: ESA-JAXA BepiColombo/MMO, ESA's Exomars, Marcopolo-R, GAIA and Jupiter Icy Satellites Explorer (JUICE). As a preparatory stage for current and future space missions, several experiments have been conducted using ESA's Venus Express and Mars Express spacecraft, Glonass satellites and Radio Astron telescope.

\section{OBSERVATIONS}

The relative position of a spacecraft can be determined by observing the spacecraft and a known near-by radio source. The detected fringe phase values from the reference source are then applied to the spacecraft. The best results are obtained when source and the target are in the same antenna beam [2]. This technique is known as VLBI phase referencing and it allows us to estimate the position of a spacecraft with an accuracy of several meters by using VLBI radio telescopes.

The spacecraft observations are conducted using standard VLBI equipment, in order to record the broadband signal emitted by the cosmic source and the narrowband carrier signal from the spacecraft. Standard spacecraft observations involve recording four frequency channels at 8 or 16 $\mathrm{MHz}$ bandwidth. These data files are later transferred electronically to JIVE for the data processing.

The data processing is conducted at JIVE using the high-resolution spectrometer software (SWSpec), the spacecraft tone tracking software (SCtracker) and finally the JIVE VLBI software correlator (SFXC) [3, 4]. The processing pipeline is summarized in the diagram of the Figure 1.

\section{APPLICATIONS}

\subsection{Ultra accurate tracking of Venus Express (VEX)}

Several VLBI tracking observations of planetary spacecraft have been conducted since 2009. The aim is to determine accurately the state vectors of the target. The major breakthrough was achieved on $28^{\text {th }}$ March 2011, when eleven radio telescopes were used to track VEX. The baselines of the array of telescopes extended $8000 \mathrm{~km}$ in East-West and $6000 \mathrm{~km}$ in North-South directions. The radio telescopes were configured to observe the spacecraft signal and the radio source (J2211-1328) in a phase-referencing mode from 08:45 to 11:30 UTC. The nodding cycle was four minutes, including 20 seconds gap for re-pointing and calibration. 


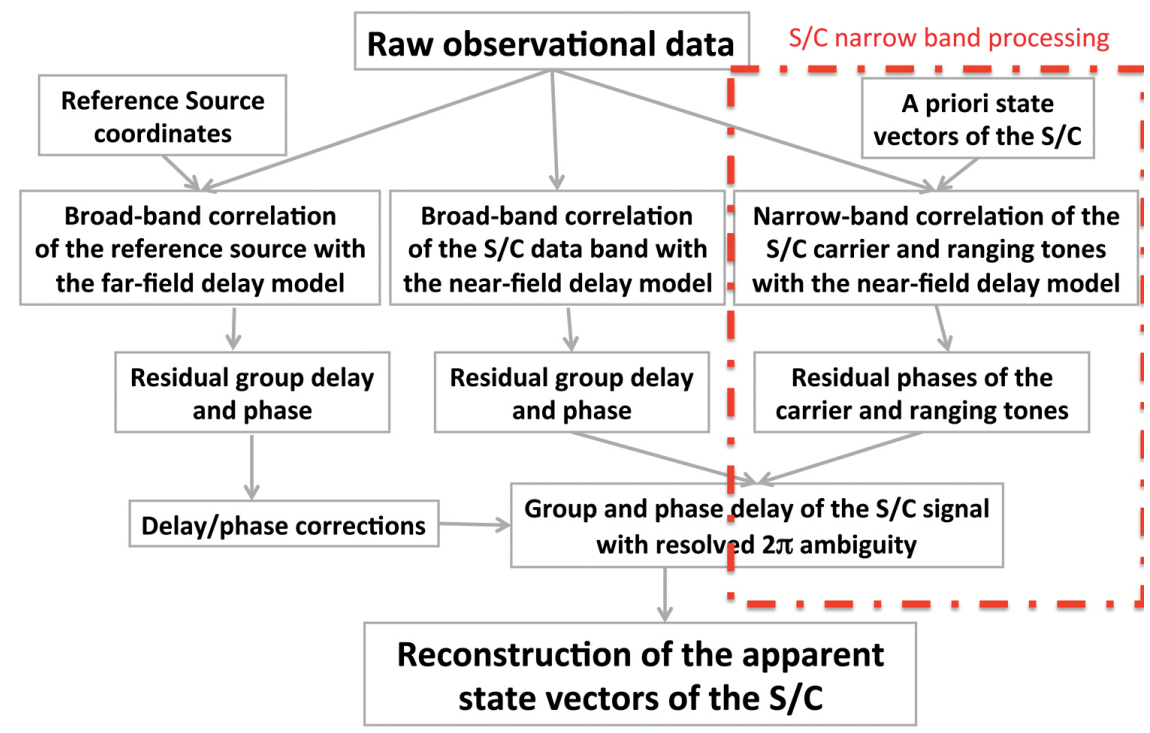

Figure 1: Block diagram with the full data flow for VLBI and Doppler tracking of spacecraft. The red box is the dedicated software for the carrier signal analysis.

The correlation and the analysis of the spacecraft signal provided residual phases of the target. These were coherently calibrated in order to image the VEX spacecraft and to derive the deviations of the lateral coordinates of the target with respect to the a priori model. The results showed an accuracy of the orbit determination of the order of 200-300 m across the track and 500-600 m along the track, as seen in Figure 2. This European VLBI Network (EVN) experiment allowed us to demonstrate that the astrometric spacecraft positioning with a very high accuracy is possible using PRIDE techniques.
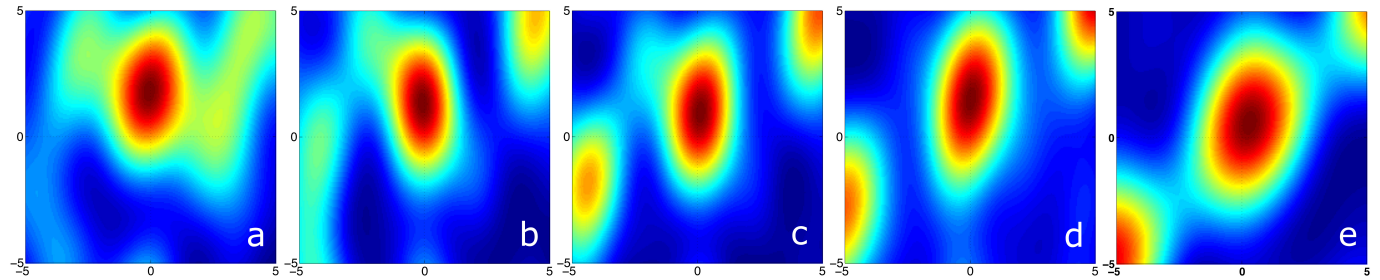

Figure 2: Radio images of VEX obtained with the EVN array. The horizontal axis represents the deviation from the nominal source Right Ascension in mas, vertical axis - deviation from the nominal source Declination in mas. a) 09:05, stations On, Wz, Ma, Mc, Mh, Sv, Zc, b) 09:30 + Ys, c) 09:55 - Sv, d) 10:20 - Mh, e) - Zc. No cleaning applied.

\subsection{Interplanetary plasma scintillations}

The solar wind is a large magnetic structure that originates on the surface of the Sun and 
expands up to hundred times the Sun's size. The structure is not homogeneous and is usually described as the sum of magnetic bubbles of different sizes and densities [5]. During the expansion strong turbulences are generated. Consequently, the radio waves that propagate within the solar wind are affected in several ways: the most well-known phenomenon is the apparent variation in the intensity and phase, which is associated to the interplanetary scintillations (IPS).

Several studies have been conducted to estimate the effect of the solar wind through the heliosphere [6]. These studies measured the intensity fluctuations of known celestial sources at low frequency. Our approach focuses on analysing the behaviour of the phase from the signal transmitted by a spacecraft. We conducted regular observations of VEX for over three years. The broad range of data allows us to characterize the fluctuations at different solar elongations and distances of the target to Earth.

By quantifying the power spectra of the phase fluctuations we retrieve the phase scintillation index $\left(\sigma_{\phi}\right)$. These were studied for over 3 years at different solar elongations and distance to the Earth. A model was built to compare our results to the theoretical Total Electron Content (TEC), as seen in Figure 3.

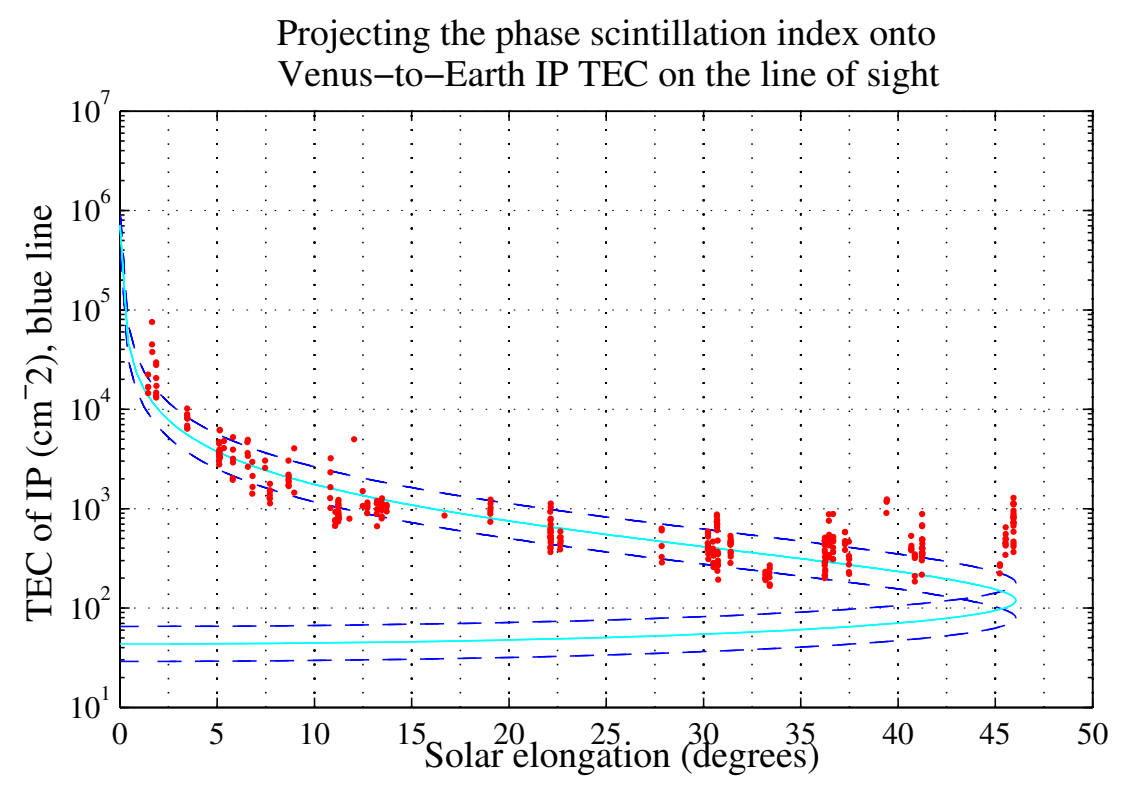

Figure 3: Superimposition of the phase scintillation index $\left(\sigma_{\phi}\right)$ and the simulated TEC (cyan) along the line of sight Venus-Earth. In dark blue, the TEC line has been multiplied by 1.5. The $\sigma_{\phi}$ values have been normalised to match with the TEC.

\subsection{Venus Express Atmospheric Drag Experiment (VExADE)}

Radio scientists have conducted several atmospheric drag experiments with VEX in order to study the upper atmosphere of Venus [7]. In these experiments the periapsis altitude of the spacecraft orbit is lowered to analyse the atmosphere above the North Pole. In this regime the spacecraft experiences the atmospheric drag, so that the trajectory is perturbed during the periapsis pass. This event is measurable with VLBI radio telescopes and the total atmospheric mass density 
at the periapsis altitude can be estimated. We conducted a preliminary drag experiment at the end of April and beginning of May. The second part of the experiment was scheduled for mid-December 2012. These included 12 consecutive days of VEX observations. The results are expected to be presented in 2013.

\subsection{Spacecraft signal as a calibrator for AGN circular polarization}

Several aspects in the configuration of Active Galactic Nuclei (AGN) are not yet well understood. Studies of the circular polarization (CP) of the radiation emitted by the AGN can contribute to understanding the jet magnetic field and the plasma configuration. VLBI imaging of the millarcsecond-scale CP offers a good approach, although it requires careful calibration.

Currently, gain transfer technique is probably the only reliable method of VLBI CP calibration, but the complexity of the observations and the data processing constrain its usage. A full experiment requires the observation of several sources over a long period of time. These sources are used to create a joint calibration curve for each element of the array. Later this gain curve is applied to each source individually. These steps decrease drastically the capacity to achieve new CP data on AGN's.

PI L. Rastorgueva proposes that our observations use a man-made calibrator: the signal transmitted by VEX spacecraft. The signal at X-band is right $\mathrm{CP}$, with leakage to left $\mathrm{CP}$ of $-32 \mathrm{~dB}$. However, the VEX signal is strong (SNR of $52 \mathrm{~dB}$ at a frequency resolution of $0.4 \mathrm{~Hz}$ with a $25-\mathrm{m}$ antenna), so the leakage is detectable with this antenna.

\subsection{Mars Express Phobos flybys}

Flybys of Phobos using the ESA's Mars Express (MEX) spacecraft are planned to be performed in 2013 and 2014. The PRIDE team, in consortium with ESPaCE (European Satelliter PArtnership for Computing Ephemerides), has proposed to collect data from the event using EVN antennas, with the objective of obtaining complementary to the radio science and astrometric data.

The radio tracking of events like this flyby are crucial to determine intrinsic parameters of planetary moons. These measurements are mainly meaningful to determine the Phobos gravity field, as well as the information on the mass and moment of inertia of Phobos. Precise determination with VLBI techniques of a spacecraft position in the vicinity of a planet is an indispensable prerequisite for interpretation of in-situ measurements.

PRIDE was already capable of detecting the MEX Phobos flyby in March 2010. In that occasion, three radio telescopes were coordinated to track the event: Yebes, Wettzell, Metsähovi. The Doppler resolution was at a level of $2-5 \mathrm{mHz}$ in $5 \mathrm{~s}$ [9].

\subsection{VLBI observation of Global Navigation Satellites}

Several tests have been conducted to analyse the radio signals emitted by GLONASS satellites with VLBI antennas [8]. These observations can open a new path for geodesy determination of positioning on Earth. The Doppler effect and the intrinsic communications signal used to track satellites are detectable in the signal processing. The broadband correlation on the calibrator allows applying phase and timing delay to the satellite results. Dual-frequency observations are recommended to improve ionospheric corrections. 
A thorough analysis of the data captured on those two experiments lead to estimate the state vectors parameters in the order of 15 to $20 \mathrm{~cm}$ accuracy [8]. We expect that increasing the number of observing telescopes, the length of the baselines and the duration of observations will lead to a significant improvement on the accuracy of the GNSS ephemerides, currently to $5 \mathrm{~cm}$. These results are expected to improve along with more observations in the near future.

\subsection{Better orbit determination of Radio Astron}

RSA's Radio Astron telescope was launched in 2011 to add a new VLBI station at the space. The telescope will introduce an extraordinary large baseline to the current network. The antenna orbits the Earth and joins regularly the VLBI observations with on-ground telescopes. The accuracy of the correlation is tightly dependent on the capabilities of the ground-station to determine its position and momenta. PRIDE is conducting observations to track the telescope and provide the estimates to the control mission.

\section{Acknowledgement}

We would like to thank the personnel of the EVN and associated radio observatories: Metsähovi (Finland), Wettzell (Germany), Medicina, Noto and Matera (Italy), Onsala (Sweden), Yebes (Spain), Hartebeesthoek (South Africa), Shanghai, Urumqi and Kunming (China), ATNF and AUSCOPE (Australia), Warkworth (New Zealand), Pushchino and KVAZAR (Russia) for their contribution to this campaign.

\section{References}

[1] J.P. Lebreton, L.I. Gurvits, et al., An overview of the descent and landing of the Huygens probe on Titan, Nature, pag. 438:758, December, 2005.

[2] Ros et al., A\&A, pag. 348-381, 1999.

[3] G. Molera Calvés, S.V. Pogrebenko, L.I. Gurvits, G. Cimo, et al., Venus Express spacecraft observations with EVN radio telescopes, Interplanetary Probe Workshop (IPPW-7), Barcelona, 2010.

[4] D.A. Duev, G. Molera Calvés, S.V. Pogrebenko, L.I. Gurvits, G. Cimo, T. Bocanegra Bahamón, Spacecraft VLBI and Doppler tracking: algorithms and implementation, A\&A, 2012.

[5] A.J. Hundhausen, A.L. Stanger, S.A. Serbicki, Mass and energy contents of coronal mass ejections: SMM results from 1980 and 1984-1988, Solar Dynamic Phenomena and Solar Wind Consequences, the third SOHO Workshop, ESA, vol. 373, pag. 409, dec 1994.

[6] A. Hewish, P.F. Scott, D. Wills, Interplanetary scintillation of small diameter radio sources, Nature, vol. 203, pag. 1214-1217, sep 1964.

[7] P. Rosenblatt et al., First ever in situ observations of Venus' polar upper atmosphere density using the tracking data of the Venus Express Atmospheric Drag Experiment (VExADE), Icarus.

[8] V. Tornatore, R. Haas, S. Casey, D.A. Duev, G. Molera Calvés, S.V. Pogrebenko, A. Keimpema, Single baseline GLONASS observations with VLBI: preliminary results, presented at $20^{\text {th }}$ EVGA meeting and analysis workshop MPIFR, Bonn, Mar 2011.

[9] G. Molera Calvés, S.V. Pogrebenko, J. Wagner, G. Cimo, L.I. Gurvits, et al., VLBI and Doppler tracking of the VEX and MEX spacecraft and future Martian missions, EPSC, Sep 2010. 